\title{
Resultados imediatos da herniorrafia inguinal com anestesia local associada com sedação ${ }^{1}$
}

\author{
Edgar Valente de Lima Neto ${ }^{2}$ \\ Alberto Goldenberg \\ Mário Jorge Jucá ${ }^{4}$
}

\begin{abstract}
Lima Neto EV, Goldenberg A., Jucá MJ. Resultados imediatos da herniorrafia inguinal com anestesia local associada com sedação. Acta Cir Bras [serial online] 2003 Set-Out;18(5). Disponível em URL: http://www.scielo.br/acb.

RESUMO - Objetivo: Avaliar os resultados imediatos da herniorrafia inguinal com anestesia local associada com sedação. Métodos: Foram operados 30 pacientes portadores de hérnia inguinal, sendo $26(86,6 \%)$ do sexo masculino e $4(13,4)$ do sexo feminino. Os anestésicos utilizados foram lidocaína a $1 \%$ e bupivacaína a $0,5 \%$ sem adrenalina, tendo sido adicionado $1 \mathrm{~mL}$ de bicarbonato de sódio para cada 9mL da solução anestésica. A sedação foi realizada com midazolan. As hérnias mais frequientes, de acordo com a classificação de Nyhus, foram as do tipo I, encontradas em $16(53,3 \%)$ pacientes. Praticou-se o reparo da parede posterior a em $17(56,6 \%)$ e em $13(43,2 \%)$ o reparo com prótese. Para a análise estatística utilizou-se o teste de McNemar para avaliar a correlação entre os escores de dor na escala E.V.A. agrupada. Resultados: Em nenhum paciente houve necessidade de mudança da técnica anestésica. Entre as complicações, observaram-se: $1(3,3 \%)$ hematoma; $1(3,3 \%)$ sangramento e $1(3,3 \%)$ infecção da ferida operatória. A deambulação foi precoce em 28 (93,3\%) pacientes, assim como a alimentação oral. O tempo de internação médio foi 18 horas, e o escore de dor na escala visual analógica (E.V.A.) foi menor ou igual a $3 \mathrm{em} 80 \%$ dos pacientes. Conclusão: A anestesia local com sedação é um método seguro, eficaz, com baixos índices de complicações imediatas, e alto índice de satisfação para pacientes selecionados, portadores de hérnia inguinal.
\end{abstract}

DESCRITORES - Hérnia inguinal. Anestesia local.

\section{Introdução}

A cada ano são praticadas cerca de 700.000 herniorrafias inguinais nos Estados Unidos da América. ${ }^{1}$ A herniorrafia inguinal é a operação mais comum no homem, atinge freqüentemente indivíduos na faixa etária em que são mais produtivos e ativos fisicamente, não raro incapacitando-os para o trabalho, com importantes implicações socioeconômicas. $^{2}$

$\mathrm{O}$ tratamento da hérnia inguinal vem evoluindo com o entendimento da anatomia, da fisiologia e da fisiopatologia. ${ }^{2}$ Kurzer, Belsham e Kark (1998) ${ }^{3}$ destacaram o desenvolvimento dos materiais de síntese e prótese. Estudos sobre o comportamento ultraestrutural das fibras de colágeno e de sua análise bioquímica foram também importantes. ${ }^{4}$ Desde Edoardo
Bassini, pioneiro da moderna cirurgia para o tratamento da hérnia, até o desenvolvimento das técnicas sem tensão, os avanços foram significativos. ${ }^{5,6}$

Observa-se grande preocupação com a discussão das opções técnicas a serem utilizadas para o tratamento da hérnia inguinal, mas nem sempre o tipo de anestesia utilizado é citado, discutido e recebe a importância que o tema merece.

O bloqueio espinhal é a técnica anestésica mais utilizada no Brasil, para a herniorrafia inguinal, o que difere do método anestésico utilizado nos vários centros de excelência no mundo. Na Clínica Shouldice, no Canadá, $95 \%$ das hérnias inguinais são operadas com anestesia local, numa experiência de mais de 230.000 operações. ${ }^{8}$ No Instituto Lichteinstein, nos Estados Unidos da América, é rotina o uso da anestesia local ${ }^{9}$.

1. Resumo da Tese de Mestrado defendida e aprovada no Programa de Pós-Graduação em Gastroenterologia Cirúrgica da Universidade Federal de São Paulo - Escola Paulista de Medicina (UNIFESP - EPM).

2. Professor Assistente da Universidade de Ciências da Saúde de Alagoas (UNCISAL) e da Universidade Federal de Alagoas (UFAL).

3. Professor Doutor, Adjunto da UNIFESP - EPM.

4. Professor Doutor, Adjunto da UFAL. 
A anestesia local para a herniorrafia inguinal não é um procedimento novo. Flanagan e Bascon (1984) $)^{10}$ descreveram relatos feitos há mais de 100 anos sobre hérnias operadas com anestesia local por Harvey Cushing, usando cocaína.

Foram descritas vantagens da anestesia local para a herniorrafia inguinal: deambulação precoce; baixos índices de tromboflebites, complicações pulmonares e de retenção urinária. ${ }^{11} \mathrm{O}$ tratamento da hérnia em regime ambulatorial está também relacionado com o tipo de anestesia. ${ }^{12}$ Callesen, Bech e Kehlet (1998) ${ }^{13}$ obtiveram baixos índices de dor e altos índices de satisfação pessoal. Outros autores relataram algumas restrições ao método: alterações anatômicas provocadas pelo intumescimento dos tecidos; relaxamento muscular inadequado; ardência; dor na infiltração dos anestésicos e contra-indicam para obesos; hérnias encarceradas; estranguladas; recidivadas e inguinoescrotais. ${ }^{2,1}$ Valdivia-Gömez, Ramirez, Sánchez, Zaga e Caneda (1994) $)^{15}$ afirmaram que o método não foi amplamente aceito por temor de não ser bem tolerado pelos pacientes. Amado $(1993)^{7}$ destacou vantagens, mas referiu que se a anestesia local for realizada de forma inadequada pode levar o paciente a vivenciar uma experiência desagradável.

Assim surgiu o questionamento: por que a anestesia local não é tão utilizada no Brasil como nos outros países? É possível que alguns cirurgiões desconheçam a técnica ou podem não estar seguros das possíveis vantagens.

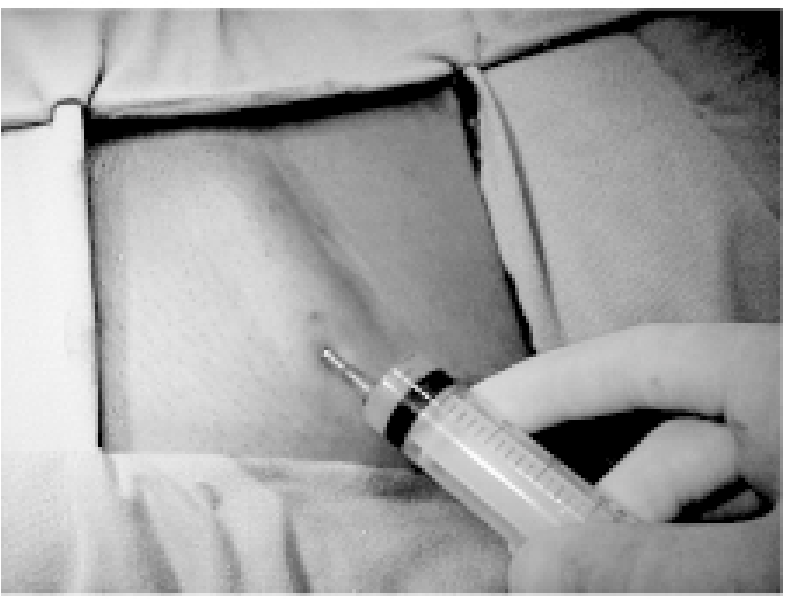

FIGURA 1 - Fotografia mostra o início da infiltração dos anestésicos.

Para sedação, empregou-se o midazolan em doses de $0,03 \mathrm{mg}$ por $\mathrm{kg}$, equivalente a $2 \mathrm{mg}$ para cada 70 $\mathrm{kg}$, administrado 10 minutos antes do início da operação por via intravenosa. Todos os atos operatórios foram
Expostos esses fatos, considerou-se oportuno observar os resultados imediatos da herniorrafia inguinal com anestesia local associada com sedação.

\section{Métodos}

\section{Amostra}

Foram estudados prospectivamente 30 pacientes portadores de hérnia inguinal. Foram excluídos pacientes com hérnia bilateral, recidivada, crural, inguinoescrotal, encarceradas e estranguladas, pacientes alérgicos aos anestésicos locais, menores de 21 anos, e aqueles que não assinaram o termo de consentimento ou não aceitaram o método. $\mathrm{O}$ projeto de pesquisa foi submetido e aprovado pela Comissão de Ética da Universidade Federal de São Paulo.

Eram 26 do sexo masculino, com média de idade de 47 anos. Apresentavam como sintomas: dor local em $56,6 \%$ dos casos; tumoração e queimação nos outros $43,4 \%$.

\section{Procedimentos}

As drogas anestésicas empregadas foram lidocaína a $1 \%$ e bupivacaína a $0,5 \%$, em partes iguais, e para cada 9 mililitros $(\mathrm{mL})$ da associação de anestésicos, foi adicionado $1 \mathrm{~mL}$ de bicarbonato de sódio. Os anestésicos não continham adrenalina. A infiltração foi realizada por planos e o bloqueio dos nervos foi feito sob visão direta (Figuras 1 e 2).

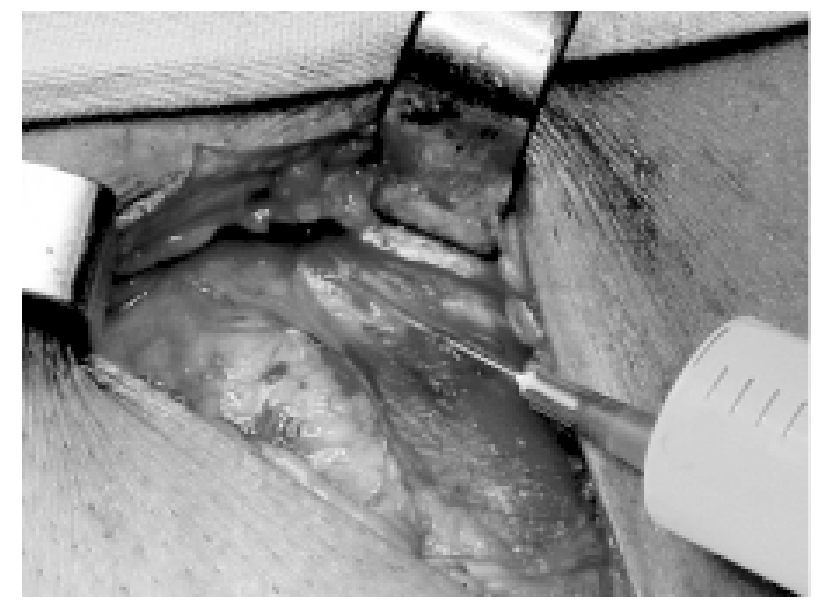

FIGURA 2 - Fotografia mostra a infiltração dos anestésicos paralela ao nervo ílio-hipogástrio.

acompanhados por anestesiologista, e todos os pacientes receberam monitorização eletrocardiográfica com aparelho Ecafix ${ }^{\circledR}$ modelo MCL com cinco derivações, e oximetria de pulso com aparelho Nellcor 
(®) modelo NT 180. A incisão foi transversa em todos os casos, e durante o ato operatório usou-se a classificação de Nyhus para se estabelecer à opção técnica de reparo. Para hérnias tipo Nyhus I e II realizou-se o reparo da parede posterior, e os pacientes que apresentaram hérnias tipo Nyhus III A e III B receberam reparo com prótese. Para o reparo da parede posterior e fixação das próteses utilizou-se o fio de náilon monofilamentar 00, e as próteses utilizadas foram de Prolene. Nos pacientes que receberam próteses, nos que tinham mais de 65 anos e nos diabéticos foi instituída a antibioticoprofilaxia com cefalotina.
Os pacientes saíram do centro cirúrgico, orientados para se alimentar e deambular quando quisessem. Foi prescrito paracetamol em doses de $750 \mathrm{mg}$ por via oral a cada 6 horas.

Procurou-se avaliar a eficácia do método anestésico. Durante o ato operatório, monitorando os sinais diretos e indiretos de dor aguda: fácies; alterações da pressão arterial; freqüência do pulso; padrão respiratório; agitação; manifestação verbal álgica; necessidade de complementação analgésica e de mudança da técnica anestésica. A escala visual analógica (EVA) foi aplicada ao término da cirurgia, com o paciente lúcido e acordado, e no momento da alta hospitalar (Quadro 1).

QUADRO 1 - Escala visual analógica ( EVA).

\begin{tabular}{l|l|l|l|l|l|l|l|l|l|l|l}
\hline 0 & 1 & 2 & 3 & 4 & 5 & 6 & 7 & 8 & 9 & 10 \\
\hline & & & & & & & & & & \\
\hline \multicolumn{1}{c}{ MULA MODIMA INTENSA }
\end{tabular}

O teste estatístico empregado foi o de McNemar, para obter a diferença estatística entre as discordâncias de dor.

\section{Resultados}

Em nenhum paciente, houve necessidade de mudança da técnica anestésica. Em 16 (53,3\%) pacientes, encontraram-se hérnias do tipo Nyhus I, e praticou-se o reparo da parede posterior em $17(56,6 \%)$ deles. Em outros $13(43,2 \%)$ o reparo herniário foi feito com prótese. Observou-se em 5 (16,6\%) pacientes bradicardia, no intra-operatório. A deambulação foi precoce em $93,3 \%$ dos pacientes, assim como o início da dieta oral, que ocorreu nas primeiras 4 horas em
$86,6 \%$ dos pacientes e sem intercorrências. As complicações pós-operatórias foram: $1(3,3 \%)$ hematoma e $1(3,3 \%)$ sangramento, sem que nenhum paciente necessitasse de reoperação. No oitavo dia de pósoperatório, quatro pacientes apresentaram edema local e um $(3,3 \%)$, infecção da ferida cirúrgica.O tempo médio de internação foi de 18 horas. A média dos escores de dor ao fim da operação foi de 2,43 (dor mínima) (Tabela 1) e de 2,53 (dor mínima) no momento da alta (Tabela 2). A correlação entre os níveis de dor, obtidos nos dois momentos, de acordo com a escala E.V.A. agrupada+, foi demonstrada na Tabela 3. Os índices de satisfação foram: bom em $66,6 \%$ e ótimo em $33,4 \%$ no momento da alta; e bom em $73,4 \%$ e ótimo em $26,6 \%$ no $8^{\circ}$ dia de pós-operatório (Tabela 4 ).

TABELA 1 - Escore de dor ao término da cirurgia.

\begin{tabular}{ccc}
\hline Escala de dor & n & $\%$ \\
\hline 0 & 3 & 10 \\
1 & 2 & 6,6 \\
2 & 6 & 20 \\
3 & 17 & 56,6 \\
4 & 2 & 6,6 \\
\hline Total & 30 & 100 \\
\hline
\end{tabular}

Média do escore de dor 2,43 (E.V.A.) = Dor mínima 
TABELA 2 - Escore de dor na alta hospitalar.

\begin{tabular}{ccc}
\hline Escala de dor & n & \% \\
\hline 0 & 3 & 10 \\
1 & 2 & 6,6 \\
2 & 7 & 23,4 \\
3 & 12 & 40 \\
4 & 6 & 20 \\
\hline Total & 30 & 100 \\
\hline
\end{tabular}

Média do escore de dor: 2,53 (E.V.A.) = Dor mínima

TABELA 3 - Correlação entre o nível de dor, de acordo com a escala EVA agrupada+, ao final da cirurgia e no momento da alta.

\begin{tabular}{c|c|c|c|c}
\hline \multirow{2}{*}{$\begin{array}{c}\text { Dor no } \\
\text { Fim da } \\
\text { Cirurgia }\end{array}$} & \multicolumn{4}{|c}{ Dor na Alta } \\
\cline { 2 - 5 } & Nula & Mínima & Moderada & Total \\
\hline Nula & 2 & 1 & 2 & 5 \\
\hline Mínima & 3 & 16 & 4 & 23 \\
\hline Moderada & - & 2 & - & 2 \\
\hline Total & 5 & 19 & 6 & 30 \\
\hline
\end{tabular}

$\%$ Concordância $=18 / 30=60,0 \%$

$\%$ Discordância $=12 / 30=40,0 \%$

Dor no fim da cirurgia maior que na alta $=5 / 30=16,7 \%$

Dor no fim da cirurgia menor que na alta $=7 / 30=23,3 \%$

Teste de McNemar $\mathrm{p}=0,774$

Não existe diferença estatisticamente significante entre as discordâncias.

TABELA 4 - Índice de satisfação pessoal na alta hospitalar.

\begin{tabular}{ccc}
\hline Índice de satisfação & $\mathbf{n}$ & $\%$ \\
\hline Ótimo & 10 & 33,4 \\
Bom & 20 & 66,6 \\
\hline Total & 30 & 100 \\
\hline
\end{tabular}

\section{Discussão}

Nos últimos anos, houve importante evolução no tratamento da hérnia inguinal. Procurou-se dar mais conforto ao doente, obter menores índices de recidiva e menor tempo de hospitalização, diminuir os custos e devolver o paciente o mais rapidamente possível às suas atividades. Com isso, obter-se-iam altos índices de satisfação.

O uso de anestésicos locais é muito freqüente nos procedimentos médico-odontológicos e, felizmente, os eventos de alergia e intolerância são muito raros. ${ }^{17}$ Neste estudo, $73,3 \%$ dos pacientes submeteram-se ao uso 
de anestésicos locais sem relatos de efeitos adversos ou alergias, apesar de 36,7\% apresentarem doenças clínicas associadas, sendo $26,7 \%$ hipertensos e $10 \%$ diabéticos.

Foi descrita a técnica de infiltração em "leque", a qual visou obter o bloqueio anestésico dos nervos mediante múltiplas punções ${ }^{18}$. É possível que essa técnica favoreça, em alguns casos, a lesão de vasos periféricos e o traumatismo de nervos. As variações são muitas, desde a forma de infiltração ao uso de anestésicos. A infiltração por planos apresenta como vantagens: a visão direta de vasos e nervos e o uso de volumes menores de anestésicos.

A lidocaína é largamente empregada na prática médica diária, é segura, possui baixa toxidade, difundese bem e rapidamente através dos tecidos; a bupivacaína possui tempo de latência longa e sua atividade é longa, sendo importante para o controle da dor no pós-operatório, pois diminui a necessidade de analgésicos opióides. ${ }^{19,20}$

A sedação com midazolan mostrou-se eficiente e pudemos constatar como é importante, pois diminui a ansiedade dos pacientes, tão comum nos procedimentos operatórios. ${ }^{9}$ A leve sonolência, determinada por doses baixas, favoreceu a realização de manobras de aumento da pressão intra-abdominal para se testar o reparo herniário intra-operatório. ${ }^{21,22}$ Outros autores destacaram os benefícios da sedação, pois esta aumenta a tolerância ao método e proporciona conforto. ${ }^{25}$

A escala visual analógica (E.V.A.) é a mais indicada para a mensuração da dor em adultos, porque é simples, rápida e objetiva. Outros métodos, como o proposto por McGill e o inventário de dor de Wiscosin, são complexos e de difícil aplicação. ${ }^{23}$ Após o término do ato operatório, os escores de dor obtidos foram baixos, mostrando a eficácia do método, pois $93,2 \%$ dos pacientes indicaram escores iguais ou abaixo de três. A segunda avaliação da dor foi realizada na alta hospitalar, quando $80 \%$ dos pacientes assinalaram escores abaixo de três. Callesen e col. ${ }^{13}$ destacaram que o diagnóstico, a avaliação e o tratamento da dor são importantes não só por motivos humanitárias e éticos, mas também por implicações econômicas, pois a dor pode prolongar a convalescença, retardando a alta e o início das atividades laborativas, e observaram, em estudo prospectivo de 466 pacientes submetidos a herniorrafia inguinal com anestesia local, que os escores de dor foram maiores no primeiro dia de pós-operatório, e que diminuíam a partir do segundo dia. Esses escores aumentaram quando o paciente se mobilizava ou tossia e foram maiores nos jovens.

A dor pós-operatória está também associada à escolha dos anestésicos. A bupivacaína é importante para a obtenção de baixos escores de dor. ${ }^{19,20}$ Outros aspectos são importantes para a diminuição da dor pósoperatória: a preservação dos nervos para eliminar o risco de neuralgia crônica; o manuseio delicado dos tecidos; a ausência de tensão do reparo herniário; a instilação de 5 a $7 \mathrm{ml}$ de solução anestésica sobre os nervos ao final da cirurgia. ${ }^{2,7,9,24,38}$

A deambulação precoce é muito benéfica, não só na prevenção das complicações tão sobejamente discutidas na literatura, como o tromboembolismo, a retenção urinária e as pneumopatias, mas também porque pode diminuir o tempo de internação hospitalar. Observou-se que 93,3\% dos pacientes deambularam precocemente. Sem dúvida a proposta da cirurgia ambulatorial ou hospital-dia para a herniorrafia inguinal está diretamente relacionada com essa grande vantagem oferecida pela anestesia local. . $^{25,26,27,28,29}$

Todos os pacientes foram orientados a se alimentarem quando quisessem, e $86,6 \%$ o fizeram nas primeiras quatro horas. Nenhum apresentou náuseas e vômitos e nenhum necessitou de hidratação venosa. Celoria e col. (1993), ${ }^{21}$ em 164 pacientes operados com anestesia local, concluíram que a introdução da dieta oral regular precoce foi benéfica e isenta de intercorrências.

Foram observadas em dois pacientes $(6,6 \%)$ complicações pós-operatórias hospitalares que não foram correlacionadas com o método anestésico. $\mathrm{Ne}$ nhum deles necessitou de reoperação. Houve hematoma em um paciente $(3,3 \%)$ e sangramento em outro $(3,3 \%)$. O pequeno hematoma superficial foi drenado, e o sangramento na linha de sutura da pele, observado três horas após a saída do paciente do centro cirúrgico, foi controlado com curativo compressivo. Amid e col., ${ }^{9} \mathrm{em} 5.000$ herniorrafias inguinais operadas com anestesia local, obtiveram índice de $1 \%$ de hematomas; Friis ${ }^{29}$ observou 2\%; Nishiguchi e Hirakawa ${ }^{30}$ observaram hematomas em 4,5\%; Bendavid ${ }^{8}$ e Wantz ${ }^{38}$ descreveram índices de hematomas abaixo de $1 \%$. Como explicar essas variações de resultados? É possível que as variações técnicas expliquem os índices diferentes observados. Exemplo é o uso de anestésicos com e sem adrenalina. Foi descrito que o uso de anestésicos com adrenalina favorece o desenvolvimento de hematomas e sangramentos. Vasos colabados sangram após o efeito da droga. Alguns autores afirmaram que poderia se esperar a ocorrência de mais hematomas e sangramentos usando-se anestésicos com adrenalina. ${ }^{10,31}$

Importante destacar que não foi observada nenhuma das complicações usualmente relacionadas com o bloqueio espinhal: cefaléia; retenção urinária; hipotensão arterial; náuseas e vômitos, e isto foi relatado como vantagens da anestesia local. ${ }^{32,25,34,35}$ 
Finley e col., ${ }^{11}$ em estudo observacional comparativo entre 880 pacientes submetidos a herniorrafia inguinal com anestesia local e 200 submetidos a herniorrafia com anestesia geral e espinhal, obtiveram índice de $0,2 \%$ de retenção urinária para o primeiro grupo e $13 \%$ para o segundo, respectivamente. Kurzer e col. $^{3}$ não observaram nenhum caso de retenção urinária em série de 3175 pacientes, apesar de um terço deles serem aposentados e a maioria com sintomas de prostatismo.

O tempo médio de internação foi de 18 horas. Outros autores obtiveram, em 466 pacientes operados, tempo médio de internação de apenas 85 minutos, contados após a saída do centro cirúrgico. ${ }^{13}$ Constatouse que a deambulação e alimentação precoces, isentas de intercorrências proporcionadas pela anestesia local, favorecem o tratamento da hérnia em regime ambulatorial e contribuem para a diminuição dos custos hospitalares. . $^{24,27,28,37}$

Observou-se que os índices de satisfação na alta hospitalar e no oitavo dia de pós-operatório foram bons em $66,6 \%$ e $73,4 \%$ respectivamente, e nenhum paciente assinalou índice regular ou péssimo. Mesmo sendo um dado subjetivo, o índice de satisfação pessoal é o resultado de um conjunto de eventos, que servem também para validar e confirmar dados obtidos, como o índice de dor e a experiência vivenciada pelo paciente. Collado e col. ${ }^{39}$ avaliaram o índice de satisfação em 221 pacientes operados, e obtiveram $90 \%$ de pacientes plenamente satisfeitos. Callesen e col. (1998) ${ }^{13}$ destacaram índices de satisfação altos, respaldados por índices de dor baixos.

A herniorrafia inguinal com anestesia local, associada com sedação, é um método que pode trazer grandes benefícios para o paciente: menor dor; deambulação e alimentação precoces; possibilidade de operação ambulatorial ou hospital-dia; índices de satisfação elevados com baixa morbidade; é simples, eficaz, pouco invasiva, traz poucas repercussões cardíacas e pulmonares e não favorece a retenção urinária.

\section{Conclusão}

A anestesia local com sedação é método seguro, eficaz, com baixos índices de complicações imediatas e alto índice de satisfação para pacientes selecionados, portadores de hérnia inguinal.

\section{Referências}

1. Rutkow IM, Robbins AW. Aspectos demográficos classificatórios e sócio-econômicos do reparo herniário nos Estados Unidos da América. Clin Cir Am Norte 1993; 3:433-48

2. Glassow F. Inguinal hernia repair using local anesthesia. Ann R Coll Surg Engl 1984; 66:382-87.
3. Kurzer M, Belsham PA, Kark AE. The lichtenstein repair. Surg Clin Noth Am 1998; 78:1025-46.

4. Wagh PV, Read RC. Defective collagen synthesis in inguinal herniation. Am J Surg 1972; 124:819-22.

5. Bassini E. Nuovo metodo per la cura radicale dell'Ernia inguinale. Atti Cong Ass Med Ital 1887; 2:179-82.

6. Lichtenstein IL, Shulman AG, Amid PK, Montllor,MM. The tension-free hernioplasty. Am J Surg 1989; 157: 188-93.

7. Amado WJ. Anestesia na cirurgia para hérnia. Clin Cir Am Norte 1993; 3:449-62.

8. Bendavid R. Results of open techniques of hernia repair. In: Schumpelick V.; Wantz GE. Inguinal hernia repair. 1ed. St. Moritz: Basel Karger; 1995. p 379-93.

9. Amid PK, Friis E, Horeyseck, Kux,M. A multi-center experience with 6,764 Lichtenstein tension-free hernioplasties. Hernia 1999; 3:47-8.

10. Flanagan Jr. L, Bascon JU. Correção da hérnia inguinal: abordagem do paciente externo com anestesia local. Clin Cir Am Norte 1984; 2:271-82.

11. Finley RK Jr., Miller SF, Jones LM. Elimination of urinary retention following inguinal herniorrafhy. Am Surg 1991; 57:486-8.

12. Felix P, Ventadoux Y, Guerineau JM. Outpatient management patient confort and satisfation of 100 consecutive inguinal hernia treated by Shouldice procedures with under local anesthesia. Ann Chir 1999; 53:387-96.

13. Callesen T, Bech K, Andersen J, Hesselfeldt P, Roikjaer O, Kehlet H. Pain after primary inguinal herniorrafhy: influence of surgical technique. J Am Coll Surg 1999; 188:355-9.

14. Robbins AW, Rutkow IM. Hernioplastia com tela-rolha. Clin Cir Am Norte 1993; 3:529-41.

15. Valdívia-Gómez G, Medina RO, Sánchez MW, Zaga MI, Caneda MM. Comparacion entre la anestesia local y el bloqueio peridural em plastia inguinal. Rev Med IMSS 1994; 33:157-60.

16. Vilela-Lima MJ, Wainstein AJA, Prado RA. Hérnias na parede abdominal. In: Fonseca FP, e Rocha PRS. Cirurgia ambulatorial. 1ed. Rio de Janeiro: Guanabara Koogan; 1999. p.588-98.

17. Fernandes ALO, Craveiro MMC, Santiago GR. Anestesia para cirurgia ambulatorial. In: Fonseca PF, e Rocha SPR. Cirurgia ambulatorial. 1ed. Rio de Janeiro: Guanabara Koogan; 1999. p.14-49.

18. Nyhus LM. An anatomic reappraisal of the posterior inguinal wall. Surg Clin North Am 1964; 44:1305-13.

19. Roberge CW, McEwen M. The effects of local anesthesics on postoperative pain. AORN 1998; 3:529-41.

20. Pettesson N, Berggren P, Larsson M, Westman B, Hahn RG Pain relief by wound infiltration with bupivacaine or highdose ropivacaine after inguinal hernia repair. Reg Anesth Pain Med 1999; 24:569-75.

21. Celoria G, Falco E, Nardini A, Stefani R, Zappia F, Castagna V, Veo R, Pesceto M. Inguinal hernia surgery under local anesthesia: technical note and immediate results. Minerva Chir 1993; 48:733-7.

22. Siragusa N, Geraci G, Li Volsi F, Guzzino M, Modica G. The treatment of inguinal hernia in a one-day surgery protocol: our experience. Minerva Chic 2000; 44:493-97.

23. Teixeira MJ, Matos PCA. Avaliação do doente com dor. In: Teixeira MJ, Figueiró JAB. Dor: epidemiologia, fisiopatologia, avaliação, síndromes dolorosas e tratamento. 1ed. São Paulo: Editora Moreira Júnior; 2001. p.58-68.

24. Moran RM. Local anesthesia for inguinal-femoral hérnia repair. In: Schumpelick V, Wantz GE. Inguinal hernia repair. 1ed. St. Moritz: 1995. p.206-11 
25. Rodrigues Jr AJ, Speranzini MB, Rasera J, Yamamura EM, Oliveira MR. Tratamento cirúrgico das hérnias inguinais com anestesia local. Rev Paul Med 1990; 108:4-5.

26. Palumbo P, Angelici A, Berardi A, Guglielmi R, Girolami M, Tombolini P, Vietri F. Surgical treatment of inguinal hernias with local anesthesia. Rev Eur Sci Med Farmacol 1992; 14:245-8.

27. Melita P, Cucinotta E, Gorgones S, Lorenzini C, Melita G. Ambulatory treatment of inguinal hernia. Chir Ital 1996; 48:39-41.

28. Murabito R, Vecchio R, Murabito P, Torrisi V, Alongi G, Panascia E, Zanghi L. Local anesthesia in the treatment of inguinal hernia. Chir Ital 1999; 51:501-05.

29. Friis E. Tension-free herniotomy using the Lichtenstein's method: results of five years experience. Ugeskr Laeger 2000; 162:1556-9.

30. Nishiguchi Y, Hirakawa K. Day surgery for adult inguinal hernia. Nippon Geda Gakkai Zasshi 2000; 101:722-8.

31. Willhelm RO, Costi F, Maia LH, Santos LM, Laurindo CD. Hernioplastia inguinal sob anestesia local. Mom e Pespec Saúde 1989; 3:17-20.

32. Teasdale C, McCrum AM, William NB, Horton RE. A randomized controlled trial to compare local with general anesthesia for short-stay inguinal hernia repair. Ann R Coll Surg Engl 1982; 64:238-42.
33. Rodrigues Jr. AJ, Mittelstaed WE, Bevilacqua JL, Bevilacqua RG, Birolini D. Anestesia local por bloqueio de campo no tratamento ambulatorial das hérnias inguinais. Rev Col Bras Cir 1992; 19:195-8

34. Rau HM, Arlt G, Peiper C, Schupelick. Surgery of inguinal hernia with local anesthesia: technique and results of a minimal invasive procedure. Chir Univ 1998; 115:1024-6.

35. Kark AE, Kurzer MN, Belsham PA. Tree thousand one hundred seventy-five primary inguinal hernia repair: advantages of ambulatory open mesh repair using local anesthesia. J Am Coll Surg 1998; 186:447-55.

36. Finley RK, Miller SF, Jones LM. Elimination of urinary retention following inguinsl herniorrafhy. Am Surg 1991; 57:486-8.

37. Andujar PR, Canario M, Grullón G, Pedro J, Ramirez A, Guerrero C, Miguel S, Valdez AP. Herniorrafia inguinal ambulatorial bajo anestesia local versus herniorrafia en admisión bajo anestesia regional. Acta Med Dom 1992; 14:84-9.

38. Wantz GE. Experience with the tension-free hernioplasty for primary inguinal hernias in men. J Am Coll Surg 1996; 183:351-6.

39. Collado OJC, Collado CE, Gonzáles GM. Hernia inguinal com anestesia local. Rev Cuba Cir 1992; 31:110-5.

\begin{abstract}
Lima Neto EV, Goldenberg S, Jucá MJ. Immediate results of inguinal hernia repair with local anesthesia associated with sedation. Acta Cir Bras [serial online] 2003 Sept-Oct;18(5). Available from URL: http://www.scielo.br/acb.

ABSTRACT - Purpose: To present the immediate results of the inguinal hernia repair with local anesthesia associated with sedation. Methods: Thirty patients were operated on of which 26 $(86,6 \%)$ were male. The age varied from 21 to 76 , and the average was of 47 years. The anesthetics used were lidocaine and bupivacaine, and the sedation was accomplished with midazolan. Results: Among the complications was observed: 1 (3,3\%) bruising; $1(3,3 \%)$ blood; and $1(3,3 \%)$ case of infection of the operative wound. Early rise in 28 patients $(93,3 \%)$, as well as the oral feeding. The average time of internment was of 18 hours, and the pain score in the analogical visual scale (A.V.S.) was smaller or equal to 3 in 24 patients (80\%). Conclusion: The local anesthesia for the inguinal hernia repair is a safe and effective method in selected patients.
\end{abstract}

KEY WORDS - Inguinal hernia. Local anesthesia.

Correspondência:

Dr. Edgar Valente de Lima Neto

Rua Guarabú, 147

57052-390 Maceió - AL

Tel: (82)241-6188

evlimant@uol.com.br
Conflito de interesse: nenhum Fonte de financiamento: nenhuma

Data do recebimento: 11/07/2003

Data da revisão: 24/07/2003

Data da aprovação: 01/08/2003 